\title{
Highly resolved, on-demand LIFT of graphene and 2D materials and computational modelling of transfer enabling conditions (Conference Presentation) (Withdrawal Notice)
}

Mado Logotheti, Symeon Papazoglou, Dimitris Kaltsas, Filimon Zacharatos, Leonidas Tsetseris, et al.

Mado Logotheti, Symeon Papazoglou, Dimitris Kaltsas, Filimon Zacharatos, Leonidas Tsetseris, loanna Zergioti, "Highly resolved, on-demand LIFT of graphene and 2D materials and computational modelling of transfer enabling conditions (Conference Presentation) (Withdrawal Notice)," Proc. SPIE 11269, Synthesis and Photonics of Nanoscale Materials XVII, 112690I (20 May 2021); doi: $10.1117 / 12.2542815$

SPIE. Event: SPIE LASE, 2020, San Francisco, California, United States 


\section{Highly resolved, on-demand LIFT of graphene and 2D materials and computational modelling of transfer enabling conditions (Conference Presentation) (Withdrawal Notice)}

Mado Logotheti, ${ }^{1}$ Symeon Papazoglou, ${ }^{1}$ Dimitris Kaltsas, ${ }^{1}$ Filimon Zacharatos, ${ }^{1}$ Leonidas Tsetseris, ${ }^{1}$ loanna Zergioti ${ }^{1}$

${ }^{1}$ National Technical Univ. of Athens (Greece)

Proceedings Volume 11269, Synthesis and Photonics of Nanoscale Materials XVII; $112690 \mathrm{I}$

(2020) https://doi.org/10.1117/12.2542815

Event: SPIE LASE, 2020, San Francisco, California, United States

Online Publication Date: 9 March 2020

Withdrawn from Publication: 20 May 2021

Publisher's Note: This conference presentation, originally published on 9 March 2020, was withdrawn per author request. 